\begin{tabular}{|c|c|c|}
\hline 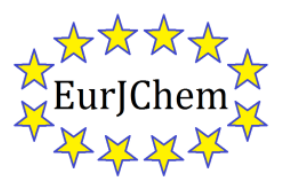 & $\begin{array}{c}\text { European Journal of Chemistry } \\
\text { Journal homepage: } \underline{\text { www.eurjchem.com }}\end{array}$ & 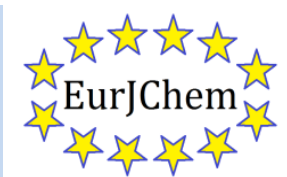 \\
\hline
\end{tabular}

\title{
Analytic perturbation theory to study the effect of screening on alignment of atomic inner shell vacancies
}

\author{
Ajay Sharma ${ }^{\mathrm{a}, *}$ and Raj Mittal ${ }^{\mathrm{b}}$ \\ a Physics Department, Chitkara University, Solan, Himachal Pradesh, IN-174103, India \\ b Physics Department, Nuclear Science Laboratories, Punjabi University, Patiala, IN-147002, India \\ *Corresponding author at: Physics Department, Chitkara University, Solan, Himachal Pradesh, IN-174103, India. Tel.: +91.172.4691800; fax: +91.172.4691800 \\ E-mail address: ajayph75@gmail.com (A. Sharma).
}

\section{COMMUNICATION INFORMATION}

\section{Received: 22 May 2010}

Received in revised form: 08 August 2010

Accepted: 08 August 2010

Online: 31 December 2010

\section{KEYWORDS}

\section{Perturbation theory}

Screening effect

Vacancy alignment

Non-relativistic dipole approximation

L sub-shells

\section{Introduction}

For photon induced atomic processes, Flügge et al. [1] were the first to explore the alignment of atomic inner shell vacancies. Calculations of vacancy alignment with different approaches have been performed by different workers, but some systematic numerical calculations were provided by Berezhko et al. [2] and Kleiman and Lohmann [3]. Up to date, the survey of literature reveals that, since eighties, the experimental measurements on alignment of photon induced $\mathrm{L} 3$ vacancies from anisotropic distribution of fluorescent X-rays in some rare earth and high $\mathrm{Z}$ elements are available from the work of eight different groups [4] those predicted contradictory results. To check the discrepancies of the results from different experimental groups and the results from experiment and theory as pointed out above, the effect of electron screening potential on the alignment calculations are undertaken in the present paper. Our earlier theoretical calculations [5] are treated as reference to observe the impact of screening on the alignment and are refined by applying screening correction [6]. In addition, an analytic perturbation theory has been developed for screened Coulomb radial wave function by interpolating the screened Coulomb potential of McEnnan et al. [7] for the elements La to $U$ in the energy region threshold to $60 \mathrm{keV}$.

\section{Formulation}

The L shell electrons being next to those in $\mathrm{K}$ shell are likely to be under screened potential. Therefore, each $\mathrm{L}$ electron seems to move in a screened Coulomb field, (Z-s).e/ $\mathrm{r}^{2}$ of the nucleus that modifies the earlier considered potential energy. L3 vacancy alignment is accounted in term of alignment parameter $A_{2}$ that is fractional difference in ionization cross- sections of L3 magnetic sub-states. The present $\mathrm{Z}$ region for which $\mathrm{A}_{2}$ has been generated is prone to screening and two different attempts have been made to study the effect of screening by;

(i) Accommodating effect of screened Coulomb potential on the earlier generated alignment parameter $A_{2}$ data [5] and

(ii) Developing an analytic perturbation theory [7] for screened Coulomb wave functions in a non-relativistic dipole approximation which, in turn, is used for the calculation of $\mathrm{A}_{2}$ for photon induced $\mathrm{L} 3$ vacancies in high $\mathrm{Z}$ elements, $57 \leq \mathrm{Z} \leq 92$.

\section{Effect of screened Coulomb potential}

To treat the $\mathrm{L}$ shell electrons under the screening nuclear pull, a screened Coulomb potential, (Z-s)e/r, of the nucleus is considered [8] instead of $\mathrm{Ze} / \mathrm{r}$ in the case of ordinary point Coulomb potential where $s$ is the screening constant. Slater [6] has suggested the rules in order to decide the value of the screening constant $\mathrm{s}$ and for a $2 \mathrm{~s}$ or $2 \mathrm{p}$ electron, $\mathrm{s}$ value comes as

$$
\mathrm{s}=2(0.85)+7(0.35)=4.15
$$

Therefore, in a screened Coulomb potential the value $a=\mathrm{Z} \alpha$ is to be modified as, $a^{*}=(Z-s) \alpha$, where $\alpha$ is fine structure constant. The modified value $a^{*}$ affects equations of formulation [5], which in turn modified single particle radial integrals $\mathrm{R}_{2}{ }^{*}$ for the bound $(\mathrm{L}=1)$ and continuum $\left(\ell^{\prime}=2\right)$ states and $\mathrm{R}_{0} *$ for bound $(\mathrm{L}=1)$ and continuum $\left(\ell^{\prime}=0\right)$ as; 


$$
\begin{aligned}
& R_{2}{ }^{*}=0.0068\left(a^{*^{5 / 2}}\right) p^{2}\left[\exp \left(1.57\left(a^{*} / p\right)\right)\right]\left|\Gamma\left(3+i\left(a^{*} / p\right)\right)\right| \times \\
& \int_{0}^{\infty}\left\{r^{6}\left[\exp \left(i p-a^{*} / 2\right) r\right] .{ }_{1} F_{1}\left[3-i\left(\frac{a^{*}}{p}\right), 6 ;(-2 i p r)\right]\right\} d r \\
& R_{0}{ }^{*}=0.2041 a^{*^{5 / 2}} p^{2}\left[\exp \left(1.57 a^{*} / p\right)\right]\left|\Gamma\left(1+i\left(a^{*} / p\right)\right)\right| \times \\
& \int_{0}^{\infty}\left\{r^{4}\left[\exp \left(i p-a^{*} / 2\right) r\right]{ }_{1} F_{1}\left[3-i\left(\frac{a^{*}}{p}\right), 6 ;(-2 i p r)\right]\right\} d r
\end{aligned}
$$

Thus, for $2 \mathrm{p}_{3 / 2}$ state, the modified alignment parameter $\left(\mathrm{A}_{2}{ }^{*}\right)$ under screened Coulomb potential is

$$
\mathrm{A}_{2} *=\frac{\left|R_{0}^{*}\right|^{2}+\frac{1}{5}\left|R_{2}^{*}\right|^{2}}{2\left|R_{0}^{*}\right|^{2}+4\left|R_{2}\right|^{2}}
$$

In this paper, the screened alignment parameter $\mathrm{A}_{2}{ }^{*}$ has been calculated for the elements $57 \leq \mathrm{Z} \leq 92$ at incident photon energy varying from $\mathrm{L} 3$ threshold to $60 \mathrm{keV}$. The calculated results are illustrated in Figure 1-5.

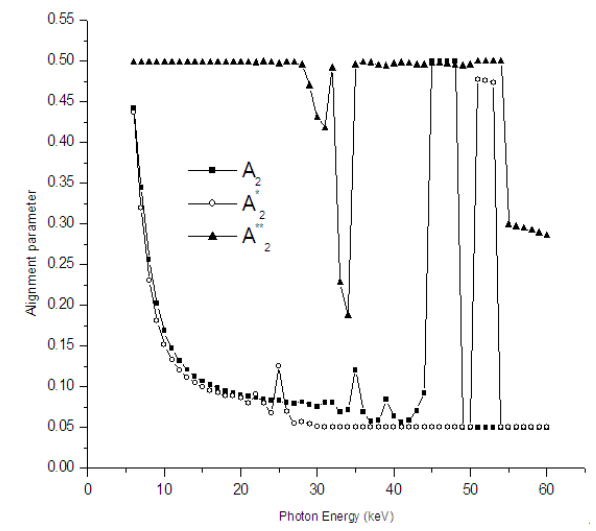

Figure 1. Plots of alignment parameter $\left(A_{2}\right)$ without screening and $\left(A_{2}{ }^{*}\right.$, $\mathrm{A}_{2}{ }^{* *}$ ) with screening at photon energies threshold to $60 \mathrm{keV}$ for La.

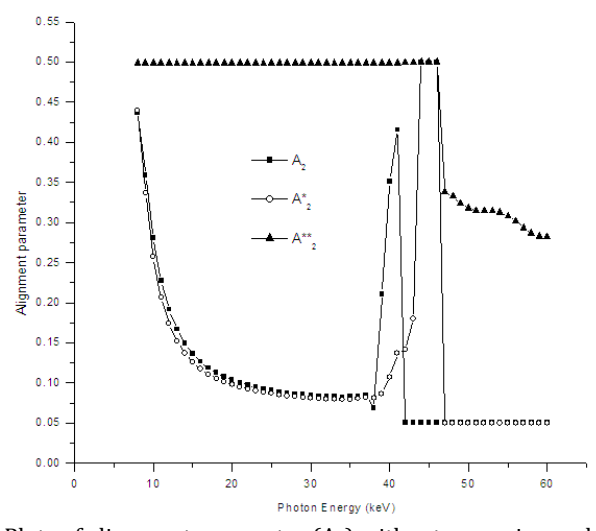

Figure 2. Plots of alignment parameter $\left(\mathrm{A}_{2}\right)$ without screening and $\left(\mathrm{A}_{2}{ }^{*}\right.$, $\mathrm{A}_{2}{ }^{* *}$ ) with screening at photon energies threshold to $60 \mathrm{keV}$ for $\mathrm{Gd}$.

\section{Analytic perturbation theory for screening effect}

For a non-relativistic radial wave function in a screened Coulomb potential, the potential inside an atom, according to analytic perturbation theory, is [7]

$$
V(r)=\left(\frac{a}{r}\right)\left[1+V_{1}(\lambda r)+V_{2}(\lambda r)^{2}+V_{3}(\lambda r)^{3}+\ldots \ldots . .\right]
$$

Here $a=Z \alpha$ and $\lambda=\alpha Z^{1 / 3}$ characterize the screening. Coefficients $V_{k}$ 's are of the order of unity. $V_{k}$ 's are alternate in sign and decrease with increasing $\mathrm{k}$ and the equation (4) converge in the region $\lambda r<1$.

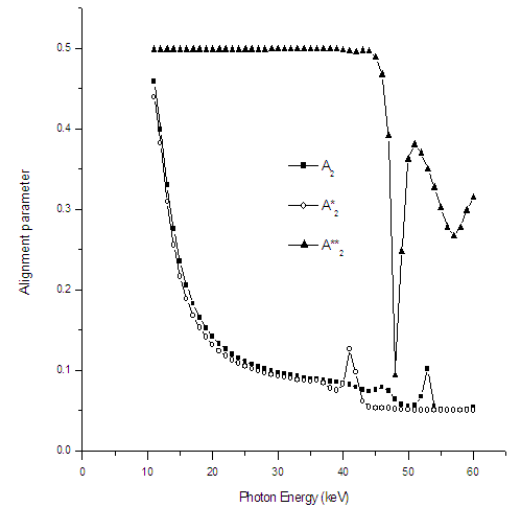

Figure 3. Plots of alignment parameter $\left(A_{2}\right)$ without screening and $\left(A_{2} *\right.$ $\mathrm{A}_{2}{ }^{* *}$ ) with screening at photon energies threshold to $60 \mathrm{keV}$ for $\mathrm{W}$.

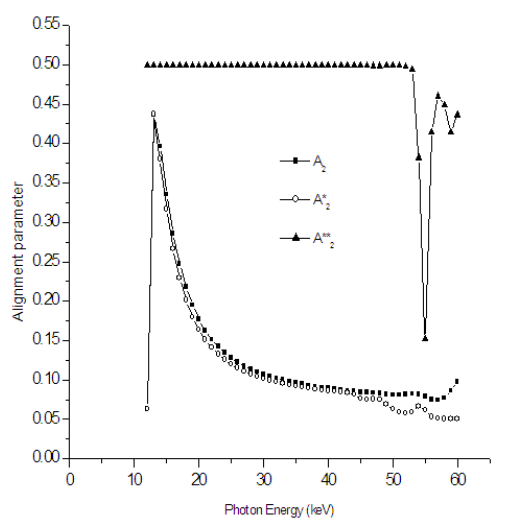

Figure 4. Plots of alignment parameter $\left(\mathrm{A}_{2}\right)$ without screening and $\left(\mathrm{A}_{2}{ }^{*}\right.$ $\mathrm{A}_{2}{ }^{* *}$ ) with screening at photon energies threshold to $60 \mathrm{keV}$ for $\mathrm{Au}$.

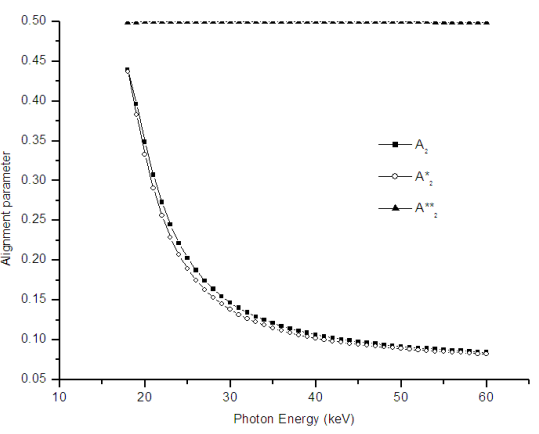

Figure 5. Plots of alignment parameter $\left(\mathrm{A}_{2}\right)$ without screening and $\left(\mathrm{A}_{2} *\right.$ $\mathrm{A}_{2}{ }^{* *}$ ) with screening at photon energies threshold to $60 \mathrm{keV}$ for Th.

In non-relativistic dipole approximation for this type of potential, the formulation [5] changes as under;

(i) Bound state wave function $\mathfrak{R}_{n \ell}(r)$ is modified as

$\mathfrak{R}^{* *}{ }_{n \ell}(r)=N_{n \ell}{ }^{* *} r^{\ell} e^{-a r / 2} S^{* *}{ }_{n \ell}(r)$

The term $S^{* *}{ }_{n_{d}}(r)$ replaces the confluent hypergeometric function used in the previous formulation [5]. Here $N^{* *}{ }_{n}$ is the modified normalization constant and for the bound state and for $2 \mathrm{p}$ state (for $\ell=1$ ), it becomes 
$N^{* *}{ }_{21}=N_{21}\left(1-30 \Lambda_{2}-320 \Lambda_{3}\right)$

where $N_{21}=\frac{(a)^{5 / 2}}{6}\left(\frac{3}{2}\right)^{1 / 2}$ is the point Coulomb normalization for the $2 p$ state and

$$
\Lambda_{k}=V_{k}(\lambda / a)^{k} \equiv V_{k}\left(1.13 Z^{-2 / 3}\right)^{k}
$$

The values of $V_{k}$ 's, for $\mathrm{k}=2$ and 3 and for the elements $\mathrm{Z}=57$ to 92, are obtained by interpolating [9] the screened Coulomb potentials of McEnnan et al. [7].

The radial wave function $S_{n \ell}^{* *}(r)$ is a polynomial in $r$ and, for $2 p$ state, it becomes

$$
S_{21}^{* *}(r)=1+\Lambda_{2}(a r)^{2}+2 \Lambda_{3}(a r)^{2}\left(3+\frac{1}{3} a r\right)
$$

Thus, the final form of the bound state wave function for $\mathrm{n}=2$ and $\ell=1$ is

$$
\begin{gathered}
\mathfrak{R}_{21}^{* *}(r)=\frac{a^{5 / 2}}{6}\left(\frac{3}{2}\right)^{1 / 2}\left[1-30 \Lambda_{2}-320 \Lambda_{3}\right] r e^{-a r / 2} \\
\left\{1+\Lambda_{2}(a r)^{2}+2 \Lambda_{3}(a r)^{2}\left(3+\frac{1}{3} a r\right)\right\}
\end{gathered}
$$

Screening also affects the binding energy of the L3 electron and the modified binding energy becomes [10]

$$
B E^{* *}=-\frac{1}{8} a^{2}\left(1+8 \Lambda_{1}+40 \Lambda_{2}+240 \Lambda_{3}\right)
$$

(ii) The continuum states $\ell^{\prime}=\ell \pm 1$ i. e. $\left(\ell^{\prime}=2\right.$ and 0$)$, the modified radial wave function is

$$
\mathfrak{R}_{p^{\prime} \ell^{\prime}}^{* *}=N_{p^{\prime} \ell^{\prime}}^{* *} r^{\ell^{\prime}} e^{-i p r} S_{\ell^{\prime}}^{* *}(p r)
$$

where $p^{\prime}$ is the magnitude of the asymptotic momentum for screened wave function [9];

$$
\begin{gathered}
p^{\prime}=p+\Delta p \quad \text { and } \Delta p=\sqrt{\frac{2\left(B E^{* *}-B E\right)}{511}} \\
N_{p^{\prime} \ell^{\prime}}^{* *}=N_{p^{\prime} \ell^{\prime}} \frac{p^{1 / 2}}{(2 \pi)^{3 / 2}}\left[\begin{array}{l}
1+\frac{1}{4} \Lambda_{2} v^{2}\left\{\begin{array}{l}
\ell^{\prime}\left(\ell^{\prime}+1\right)\left(2 \ell^{\prime}+1\right)-\left[3 v^{2}+\ell^{\prime}\left(\ell^{\prime}+1\right)+3 n^{2}-\ell(\ell+1)\right] \\
{\left[2 \ell^{\prime}+1-\rho_{\ell}\right]}
\end{array}\right\} \\
-\frac{1}{4} \Lambda_{3} v^{4}\left(\begin{array}{l}
\left.\frac{5}{3} \ell^{\prime}\left(\ell^{\prime}+1\right)\left(2 \ell^{\prime}+1\right)-\left\{\begin{array}{l}
5 v^{2}-1+3 \ell^{\prime}\left(\ell^{\prime}+1\right)-\left(n^{2} / v^{2}\right) \\
{\left[5 n^{2}+1-3 \ell(\ell+1)\right]}
\end{array}\right\}\right) \\
{\left[2 \ell^{\prime}+1-\rho_{\ell^{\prime}}\right]}
\end{array}\right]
\end{array}\right.
\end{gathered}
$$

is the screened Coulomb normalization constant [9] and

$$
N_{p^{\prime} \ell^{\prime}}=(2 p)^{\ell^{\prime}} \frac{\left|\Gamma\left(\ell^{\prime}+1+i v\right)\right|}{\Gamma\left(2 \ell^{\prime}+2\right)} e^{\pi v / 2}
$$

is the point Coulomb normalization constant for the continuum state and $v=a / p$. $\rho_{\ell^{\prime}}=\rho_{\ell^{\prime}-1}+\frac{2 v^{2}}{\ell^{\prime 2}+v^{2}}, \rho_{0}=1-\frac{2 \pi v}{e^{2 \pi v}-1}$ and $\rho_{2}=\rho_{0}+\frac{2 v^{2}}{1+v^{2}}+\frac{2 v^{2}}{4+v^{2}}$

Thus, the screened continuum radial function is

$s_{\ell^{\prime}}^{* *}(p r)=s_{\ell^{\prime}}(p r)+\lambda^{2} A_{2}\left(\ell^{\prime}, p r\right)+\lambda^{3} A_{3}\left(\ell^{\prime}, p r\right)+\ldots \ldots$.

where $s_{\ell^{\prime}}(p r)=M\left(\ell^{\prime}+1+i v, 2 \ell^{\prime}+2,2 i p r\right)$

is the usual point Coulomb radial wave function and

$$
\begin{aligned}
A_{k}\left(\ell^{\prime}, p r\right)= & \sum_{s=-k}^{k} \alpha_{s}^{k}\left(-i v, \ell^{\prime}\right) M\left(\ell^{\prime}+1+i v-s, 2 \ell^{\prime}+2,2 i p r\right)+ \\
& \beta_{0}^{k}\left(-i v, \ell^{\prime}\right) \frac{\partial}{\partial v} M\left(\ell^{\prime}+1+i v, 2 \ell^{\prime}+2,2 i p r\right)
\end{aligned}
$$

Here $M(f, g, z)={ }_{1} F_{1}(f, g, z) / \Gamma(g)$ is the regular confluent hyper-geometric expression and is evaluated from its series expression with argument parameters $\mathrm{f}, \mathrm{g}$ and $\mathrm{z}$ as [11]

${ }_{1} F_{1}(f ; g ; z)=\left\{\sum_{k=0}^{20}\left\{\frac{(f+k-1) !}{(f-1) !} / \frac{(g+k-1) !}{(g-1) !}\right\} \frac{z^{k}}{k !}\right\} / \Gamma(g)$

(ii) a. For the continuum state $\ell^{\prime}=2$, the normalization constant becomes [9]

$$
\begin{aligned}
& N_{p^{\prime} 2}^{* *}=\frac{p^{1 / 2}(2 p)^{2}}{(2 \pi)^{3 / 2}} \frac{|\Gamma(3+i v)|}{\Gamma(6)} e^{\pi v / 2}\left[\begin{array}{l}
1+0.25 \Lambda_{2} v^{2}\left\{30-\left[3 v^{2}+16\right]\left[5-\rho_{2}\right]\right\} \\
-0.25 \Lambda_{3} v^{4}\left(\begin{array}{l}
50-\left\{5 v^{2}+17-4 / v^{2}[15]\right\} \\
{\left[5-\rho_{2}\right]}
\end{array}\right]
\end{array}\right] \\
& S_{2}(p r)=M(3+i v, 6,2 i p r)
\end{aligned}
$$

and

$$
\begin{aligned}
A_{2}(2, p r)= & \frac{v^{2} V_{2}}{4 a^{2}}\left[\begin{array}{l}
\left(21 v^{2}+60\right) M(3+i v, 6,2 i p r)+((3-i v) 2 i v(2 i v-1)-10) \\
M(2+i v, 6,2 i p r)+(0.5 i v(4-i v)(3-i v)) M(1+i v, 6,2 i p r)+(3+i v) \\
{[-2 i v(-2 i v-1)-10] M(4+i v, 6,2 i p r)-0.5 i v(4+i v)(3+i v) M(5+i v, 6,2 i p r)}
\end{array}\right] \\
& +\frac{4 v\left(v^{2} V_{2}\right)}{4 a^{2}}\left\{0.5\left[3 v^{2}+6\right]+5\right\} \frac{\partial}{\partial v} M(3+i v, 6,2 i p r)
\end{aligned}
$$

Similarly,

$$
\begin{aligned}
A_{3}(2, p r)= & \frac{v^{4} V_{3}}{4 a^{3}}\left[\begin{array}{l}
-\left(40 v^{2}+52+360 / v^{2}\right) M(3+i v, 6,2 i p r)+\left(0.5(3-i v)(15 i v)(1-i v)+12-120 / v^{2}\right) \\
M(2+i v, 6,2 i p r)+(1.5(1-i v)(4-i v)(3-i v)) M(1+i v, 6,2 i p r)-1 / 6(5-i v)(4-i v) \\
(3-i v) M(i v, 6,2 i p r)+\left(0.5(3+i v)(-15 i v)(1+i v)+12-120 / v^{2}\right) M(4+i v, 6,2 i p r) \\
+1.5(1+i v)(4+i v)(3+i v) M(5+i v, 6,2 i p r)-1 / 6(5+i v)(4+i v)(3+i v) \\
M(6+i v, 6,2 i p r)
\end{array}\right] \\
+ & \frac{2 v\left(v^{4} V_{3}\right)}{4 a^{3}}\left\{-5 v^{2}-17+60 / v^{2}\right\} \frac{\partial}{\partial v} M(3+i v, 6,2 i p r)
\end{aligned}
$$

Thus, the screened continuum radial function for the state $\ell^{\prime}=2$ becomes

$s_{2}^{* *}(p r)=M(3+i v, 6,2 i p r)+\lambda^{2} A_{2}(2, p r)+\lambda^{3} A_{3}(2, p r)+\ldots .$.

and the final form of the continuum wave function is

$$
\Re_{p 2}^{* *}\left(p^{\prime} r\right)=N_{p^{\prime} 2}^{* *} r^{2} e^{-i p r} s_{2}^{* *}(p r)
$$


(ii) b. Similarly, for the continuum state $\ell^{\prime}=0$, the normalization constant becomes [9];

$$
N_{p^{\prime} 0}^{* *}=\frac{p^{1 / 2}}{(2 \pi)^{3 / 2}} \frac{|\Gamma(1+i v)|}{\Gamma(2)} e^{\pi v / 2}\left[\begin{array}{l}
1+0.25 \Lambda_{2} v^{2}\left\{-\left[3 v^{2}+10\right]\left[1-\rho_{0}\right]\right\} \\
-0.25 \Lambda_{3} v^{4}\left(\begin{array}{l}
\left.-\left\{5 v^{2}-1-4 / v^{2}[15]\right\}\right) \\
{\left[1-\rho_{0}\right]}
\end{array}\right]
\end{array}\right]
$$

$$
s_{0}(p r)=M(1+i v, 2,2 i p r)
$$

and

$$
\begin{aligned}
A_{2}(0, p r)= & \frac{v^{2} V_{2}}{4 a^{2}}\left[\begin{array}{l}
\left(9 v^{2}+20\right) M(1+i v, 2,2 i p r)+((1-i v) 2 i v(2 i v-1)-10) \\
M(i v, 2,2 i p r)+0.5 i v(2-i v)(1-i v) M(-1+i v, 2,2 i p r)+(1+i v) \\
{[-2 i v(-2 i v-1)-10] M(2+i v, 2,2 i p r)-0.5 i v(2+i v)(1+i v) M(3+i v, 2,2 i p r)}
\end{array}\right] \\
& +\frac{4 v\left(v^{2} V_{3}\right)}{4 a^{2}}\left\{0.5\left[3 v^{2}\right]+5\right\} \frac{\partial}{\partial v} M(1+i v, 2,2 i p r)
\end{aligned}
$$

Similarly,

$$
\begin{aligned}
A_{3}(0, p r)=\frac{v^{4} V_{3}}{4 a^{3}}\left[\begin{array}{l}
-\left(20 v^{2}+2+120 / v^{2}\right) M(1+i v, 2,2 i p r)+\left(0.5(1-i v)(15 i v)(1-i v)-6-120 / v^{2}\right) \\
M(i v, 2,2 i p r)+(1.5(1-i v)(2-i v)(1-i v)) M(-1+i v, 2,2 i p r)-1 / 6(3-i v)(2-i v)(1-i v) \\
M(-2+i v, 2,2 i p r)+\left(0.5(1+i v)(-15 i v)(1+i v)-6-120 / v^{2}\right) \\
M(2+i v, 2,2 i p r)+1.5(1+i v)(2+i v)(1+i v) M(3+i v, 2,2 i p r)-1 / 6(3+i v)(2+i v) \\
(1+i v) M(4+i v, 2,2 i p r)
\end{array}\right] \\
+\frac{2 v\left(v^{4} V_{3}\right)}{4 a^{3}}\left\{-5 v^{2}+1+60 / v^{2}\right\} \frac{\partial}{\partial v} M(1+i v, 2,2 i p r)
\end{aligned}
$$

Therefore, the screened continuum radial function for the state $\ell^{\prime}=0$ becomes

$$
s_{0}^{* *}(p r)=M(1+i v, 2,2 i p r)+\lambda^{2} A_{2}(0, p r)+\lambda^{3} A_{3}(0, p r)+\ldots . .
$$

and the final form of the continuum wave function for $\ell^{\prime}=0$

$$
\Re_{p 0}^{* *}\left(p^{\prime} r\right)=N_{p^{\prime} 0}^{* *} e^{-i p r} s_{0}^{* *}(p r)
$$

The single particle radial integral for bound $(\mathrm{L}=1)$ and continuum $\left(\ell^{\prime}=2\right)$ state reduces as

$$
R_{\text {matrix } 2}^{* *}=\int_{0}^{\infty} r^{3}\left[\Re_{2}^{* *}\left(p^{\prime} r\right)\right]^{*}\left[\Re_{21}^{* *}(r)\right] d r
$$

Similarly, for $\mathrm{L}=1$ and $\ell^{\prime}=0$ state

$$
R_{\text {matrix } 0}^{* *}=\int_{0}^{\infty} r^{3}\left[\Re_{0}^{* *}\left(p^{\prime} r\right)\right]^{*}\left[\Re_{21}^{* *}(r)\right] d r
$$

Thus, for $2 \mathrm{p}_{3 / 2}$ state the screened alignment parameter

$$
\mathrm{A}_{2} *=\frac{\left|R_{\text {matrix } 0}^{* *}\right|^{2}+\frac{1}{5}\left|R_{\text {matrix } 2}^{* *}\right|^{2}}{2\left|R_{\text {matrix } 0}^{* *}\right|^{2}+4\left|R_{\text {matrix } 2}^{* *}\right|^{2}}
$$

To check the authenticity of the present method of calculation of $\mathrm{A}_{2}{ }^{* *}$, the intermediate steps are compared with our earlier formulation [5] of point Coulomb calculations. A close agreement has been obtained among the values of intermediate steps involved in both the formulations that proved the reliability of these calculations and prompted us to extend our calculations for elements La to $U$ in the energy region threshold to $60 \mathrm{keV}$. The calculations are performed in mathematica 5.1 and the results are illustrated in Figure 1-5.

\section{Results and discussion}

The results show that screening has no effect on the shape of plots of $\mathrm{A}_{2}{ }^{*}$ at the lower end of energies but the values are lowered by 4 to $10 \%$ of the earlier values. For the elements $57 \leq \mathrm{Z} \leq 79$ in the energy region 43 to $55 \mathrm{keV}$ like the earlier results [5] on $\mathrm{A}_{2}$, again the peak structures in $\mathrm{A}_{2}{ }^{*}$ plots appear. For elements $57 \leq \mathrm{Z} \leq 63$ the peak structure is distorted and the gap between two groups of peaks increases, one group shifts comparatively to lower energy and other shifts to higher energy side (Figure 1). For the elements $64 \leq \mathrm{Z} \leq 73$, the peak shifting is less but the peak becomes more prominent as compared to the case of without screening (Figure 2). The peak structure in Figure 3 for $\mathrm{W}$ shows the pattern similar to shifting of lower group of peaks in case of La and the trend remains the same up to Pt. For Au slight peak structure in $\mathrm{A}_{2}{ }^{*}$ appears at the high energies that was almost absent for $A_{2}$ in the same energy region (Figure 4).

Similar $\mathrm{A}_{2}{ }^{*}$ peaks, though low in intensity, appear for elements $\mathrm{Z}=81$ to 83 . All this confirms that peak structures have shifted to lower energy side. For elements $\mathrm{Z}>83$ there is smooth fall in $\mathrm{A}_{2} *$ with energy (Figure 5). The peak structures may be due to some disturbance of the point Coulomb potential with the starting of the $\mathrm{K}$ shell ionization and the explanation [5] still holds with screening effects though there is shift in energy.

Alignment parameter $\mathrm{A}_{2}^{* *}$ takes maximum value $(\sim 0.5)$ in the regions starting from L3 threshold energy to about 30 to 45 $\mathrm{keV}$ for all the elements. The range shifts to higher energy side for increasing Z's. For the elements with Z varying from 57 to 70 a decrease in the alignment value exists at $\sim 30 \mathrm{keV}$ (Figure 1-4).This downfall in the alignment also increases with Z's and attains its least value $\sim 0.05$ for the most of the elements. Moreover, this down fall is in the form of peak structure in the energy region 40 to $50 \mathrm{keV}$. The alignment remains almost constant $(\sim 0.5)$ for all the high $\mathrm{Z}$ elements in the range 81 to 92 with a very small variation at the third decimal place throughout whole energy range (Figure 5). The alignment parameter values being $>0.1$ at energies $<20 \mathrm{keV}$ are certainly higher than 5 to $8 \%$ uncertainties quoted in experimental results. The presence of energy pockets where the alignment is comparatively prominent requires further exploration either with the precise measurements of low intensity $\mathrm{L} x$-rays $\left(\mathrm{L}_{\ell}\right.$ and $\mathrm{L}_{\alpha 2}$ ) or by modifications of theoretical calculations. In Figures $1-5, A_{2}^{* *}$ looks just as reflection or complement of variations in $\mathrm{A}_{2}$ and $\mathrm{A}_{2}{ }^{*}$ values. Therefore, the explanation for the peak structure [5] may still be appreciable for the screening effect derived through analytical perturbation theory.

\section{References}

[1]. Flügge, S.; Mehlhorn, W.; Schmidt, V. Phys. Rev. Lett. 1972, 29, 7-9.

[2]. Berezhko, E. G.; Kabachnik, N. M.; Rostovsky, V. S. J. Phys. B. 1978, 11 1749-1758.

[3]. Kleiman, U.; Lohmann, B. J. Elect. Spectro. Rel. Phen. 2003, 131, 29-50.

[4]. Sharma, A.; Mittal, R. N. Instr. Meth. Phys. Res. A. 2010, 619, 55-58.

[5]. Sharma, A.; Singh, M.; Mittal, R. Pramana 2006, 66, 1111-1118.

[6]. Slater, J. C. Phys. Rev. 1930, 36, 57-65.

[7]. McEnnan, J.; Kissel, L.; Pratt, R. H. Phys. Rev. A 1976, 13, 532-558.

[8]. Hall, H. Rev. Mod. Phys. 1936, 8, 358-359.

[9]. Sharma, A.; Mittal, R. Vacancy alignment in atomic inner shells, Lambert Academic Publishing, 2010.

[10]. Oh, S. D.; McEnnan, J.; Pratt, R. H. Phys. Rev. A. 1976, 14, 1428-1436

[11]. Wolfram, S. The Mathematica, Cambridge University Press, 1996. 